\title{
Pelvic pain in endometriosis: is success of therapy gone in cigarette smoke?
}

\author{
Agnes Koppan $^{1^{*}}$, Judit Hamori ${ }^{2}$, Ildiko Vranics ${ }^{1}$, Janos Garai $^{3}$, Ildiko Kriszbacher ${ }^{1}$, \\ Jozsef Bodis ${ }^{2}$, Frank Oehmke ${ }^{4}$, Hans-Rudolph Tinneberg ${ }^{4}$, Miklos Koppan ${ }^{2,4}$ \\ ${ }^{1}$ Institute of Nursing and Clinical Sciences, Faculty of Health Sciences, University of Pécs, Pécs, Hungary; \\ *Corresponding Author: akoppan@gmail.com \\ ${ }^{2}$ Department of Obstetrics and Gynecology, Faculty of Medicine, University of Pécs, Pécs, Hungary; \\ ${ }^{3}$ Department of Pathophysiology and Gerontology, Faculty of Medicine, University of Pécs, Pécs, Hungary; \\ ${ }^{4}$ Department of Obstetrics and Gynecology, Faculty of Medicine, University of Giessen, Giessen, Germany.
}

Received 17 January 2011; revised 1 June 2011; accepted 10 August 2011.

\section{ABSTRACT}

The objective of the study was to assess potential individual factors influencing the efficacy of combined surgical and medical therapy in endometriosis patients with pelvic pain. For this purpose we performed a prospective study using a specifically designed questionnaire among patients suffering from persistent pelvic pain and undergoing laparoscopy and further $\mathrm{GnRH}$ analogue therapy in a university-based gynecologic department. Eighty-one women of reproductive age with histologically confirmed endometriosis were enrolled. A questionnaire gathered information from women on the following groups of variables: age, marital status, education, reproductive and medical history including previous pregnancies and parity, knowledge of accompaniing pelvic disorders, concurrent cigarette smoking, as well as general quality of life estimates including self-image. Pelvic pain was scored using a visual analogue scale. Patients filled out the questionnaires before surgery and upon completing medical therapy. Data were statistically evaluated. After cessation of therapy, $53 \%$ of patients reported absence of pain. Only $12 \%$ of pain-free patients were smokers. This corresponded to slightly more than one third $(35 \%)$ of all smokers in the study. However, $56 \%$ of non-smoker participants reported a positive outcome that proved to be significantly larger than the ratio of pain-free smoker participants $(p=0.02)$. Improvement in quality of life was reported by $74 \%$ of all patients, and only $9 \%$ of them were smokers. However, $47 \%$ were smokers among patients reporting no change or worsening in quality of life $(p<0.01)$. Based on our results, we can conclude, that regular smoking might have a disadvantageous impact on the success rate of combined surgical and medical therapy for endometriosis related pelvic pain.

Keywords: Pelvic Pain; Endometriosis; Cigarette Smoke

\section{INTRODUCTION}

Endometriosis affects millions of women world wide. It can severely alter quality of life and leads to extensive problems with fertility and loss of work time [1]. Endometriosis might remain asymptomatic and discovered accidentally. However, it may cause symptoms, which include chronic pelvic pain, bleeding, infertility, and increases susceptibility to development of adenocarcinoma [2]. Signs and symptoms arise from cyclic bleeding into the surrounding tissues, resulting in inflammation and formation of scarring and adhesions. It is peculiar, that symptom severity does not correlate well with the extent or progression of the lesions [3]. Minor laparoscopic findings might come with severe complaints, while extensive lesions might remain undetected and revealed only accidentally. The exact roles of different factors contributing to the establishment and persistence of the endometriotic lesion are still not fully understood. Despite the high associated morbidity and health care costs, the incidence, prevalence, and risk factors of endometriosis remain uncertain.

Symptomatic endometriosis can be managed surgically and/or medically. The aim is pain relief and/or amelioration of infertility. Medical treatment is usually long term, and recurrence is frequent after its cessation. Classic endometriosis pharmacotherapy is represented 
by GnRH agonists, oral contraceptives and Type II progesterone receptor ligands [4]. All medical treatments seem to be equally effective in managing endometriosis. Although about $80 \%$ - $85 \%$ of patients have improvement in their symptoms [5], many women experience unsatisfactory results. However, little is known about factors on patient's side influencing the efficacy of generally accepted therapeutic approaches used to alleviate symptoms caused by endometriosis.

In our study, we investigated the effectiveness of combined surgical and medical therapy of patients with histologically confirmed endometriosis with regard to pain relief and overall quality of life issues. To determine these parameters we used a questionnaire before laparoscopic surgery and upon cessation of post-surgery medical therapy. With that, we determined efficacy of therapy from the patient's point of view, with relation to several non-medical variables such as marital status, level of education and smoking.

\section{MATERIALS AND METHODS}

\subsection{Study Population and Sample}

The prospective cohort study population consisted of patients of reproductive age complaining about persistent pelvic pain and undergoing laparoscopy in our departments (Department of Obstetrics and Gynecology, Faculty of Medicine, University of Pécs, Hungary and Department of Obstetrics and Gynecology, Faculty of Medicine, University of Giessen, Germany). Following laparoscopy and histological examination, a random sample of 150 patients with histologically proven endometriosis were then recruited. This initial number of recruited patients was arbitrarily set and reached in a 6 month period between June and December, 2008. Randomization was based on the unpaired character of patients' social security number. The refusal rate upon reaching the desired number of 150 was less than 5\%, however, the drop-out rate during the entire study period was $46 \%$. Those who were lost for follow-up did not differ in any characteristics based on the collected data comparing to those who completed both questionnaires. Final statistics were carried out using data from those 81 patients completing the study. Prior to the operation patients consented to participate in the study. A standardized questionnaire elicited information from women on the following groups of variables: age, marital status, education, reproductive and medical history. The questionnaire was purposefully designed to ascertain information on potential confounders, which included gravidity (number of pregnancies regardless of outcome) and parity (number of live births), knowledge of accompaniing pelvic disorders, concurrent cigarette smoking and caffein intake, since all have been reported as risk factors for endometriosis [6,7]. Further variables were concurrently used medication including pain killers, as well as daily habits of excersice, type of work and general quality of life estimates including self-image. Pelvic pain was scored using a visual analogue scale from 0 - 10 . Only patients with histologically confirmed endometriosis and with no other pelvic/abdominal alteration or disease confirmed at laparoscopy were then eligible to continue the study. Patients then received a 6 month GnRH analogue therapy and were asked to fill out the same questionnaire upon completing medical therapy. The number of eligible patients completing both questionnaires was 81 .

\subsection{Operative Procedures}

Laparoscopies were performed by highly trained and experienced surgeons. Following the operations they completed a standardized operative report to ascertaine information on postoperative diagnosis and other pathology regardless of surgical indication. Severity of endometriosis was staged according to the American Fertility Society's revised definition. In all patients endometriosis lesions were laparoscopically removed and/ or electrocauterized and histological examination confirmed diagnosis. The affiliated University gave Institutional Review Board approval for the conduct of this study.

\subsection{Statistical Analysis}

Analysis of data was performed using Microsoft Excel and SPSS 15 programs. We applied chi-square test, analysis of variance (ANOVA), and Pearson-Spearman's rank correlation test. Data are presented as percentage values.

\section{RESULTS}

Mean age of participating patients was 31.2 years $(21$ - 43 years, SD = 5.24). Out of them, $17.2 \%$ were regular smokers.

\subsection{Outcomes in Pain Relief}

At the end of the treatment period, $53 \%$ of patients reported the total absence of pain that they had specifically complained about at the beginning of the study. Only $12 \%$ of pain-free patients were smokers. This means that slightly more than one third (36\%) of all smokers, while $57 \%$ of all non-smoker participants reported a positive outcome in the study. This difference proved to be significant, as calculated by Chi-Square test $(p=0.02$, Figure 1). 


\subsection{Outcomes in Overall Quality of Life (Self-Image)}

Overall, 74\% of patients reported improvement in their general quality of life, while no improvement or even deterioration was reported by $26 \%$ of all participants. Among those with improved quality of life only $9 \%$ were smokers, while $47 \%$ were smokers among patients reporting no change or even worsening in their quality of life. That means, only $36 \%$ of all smokers in the study reported improvement in their quality of life, while $64 \%$ of them reported no change or worsening.
The correlation between smoker status and negativ quality of life outcome proved to be significant, as calculated by the Pearson's correlation test (2-tailed, $p<0.01$, Figure 2).

Detailed analyses revealed no significant differences in pain relief and quality of life measures at the end of therapy with relation to marital status, level of education, number of previous pregnancies regardless of outcome and parity (number of live births). Furthermore, analyzing the data concerning the extent of the disease (i.e. the stage of endometriosis recorded at laparoscopy) and pain scores and quality of life values at the beginning and the

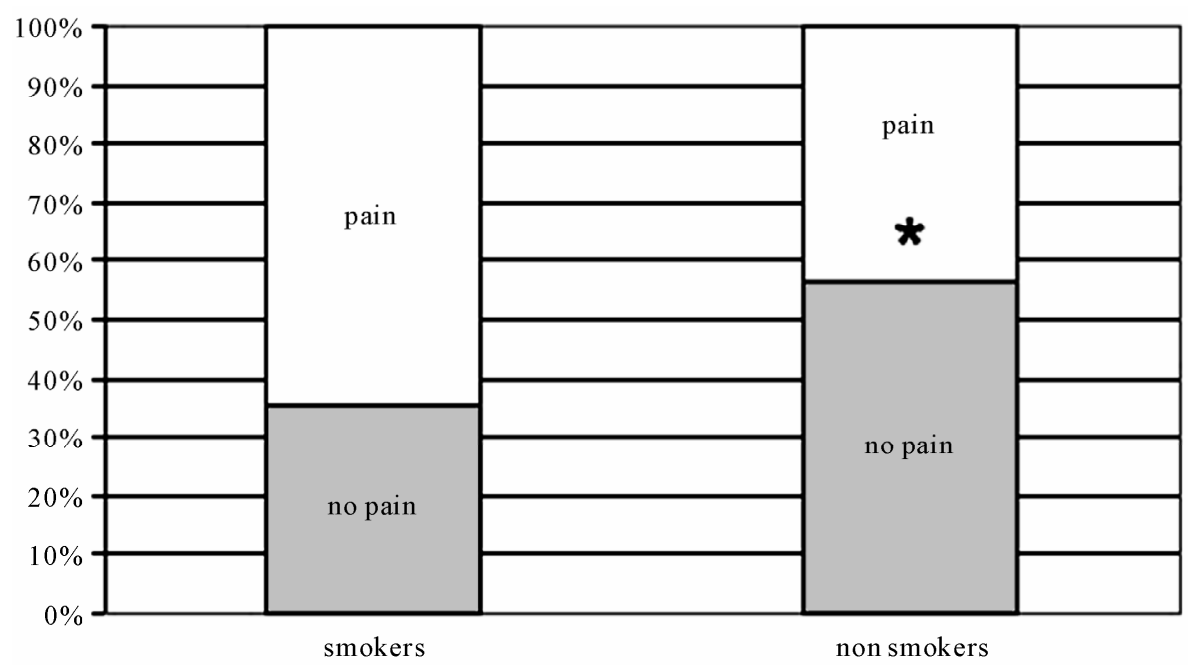

Figure 1. Correlation between smoker status and absence of specific pain as a basis for complaints after combined surgical and medical therapy of endometriosis patients. Data are presented as percentage values. ${ }^{*} p=0.02$; pain, specific pain is present; no pain, specific pain is absent.

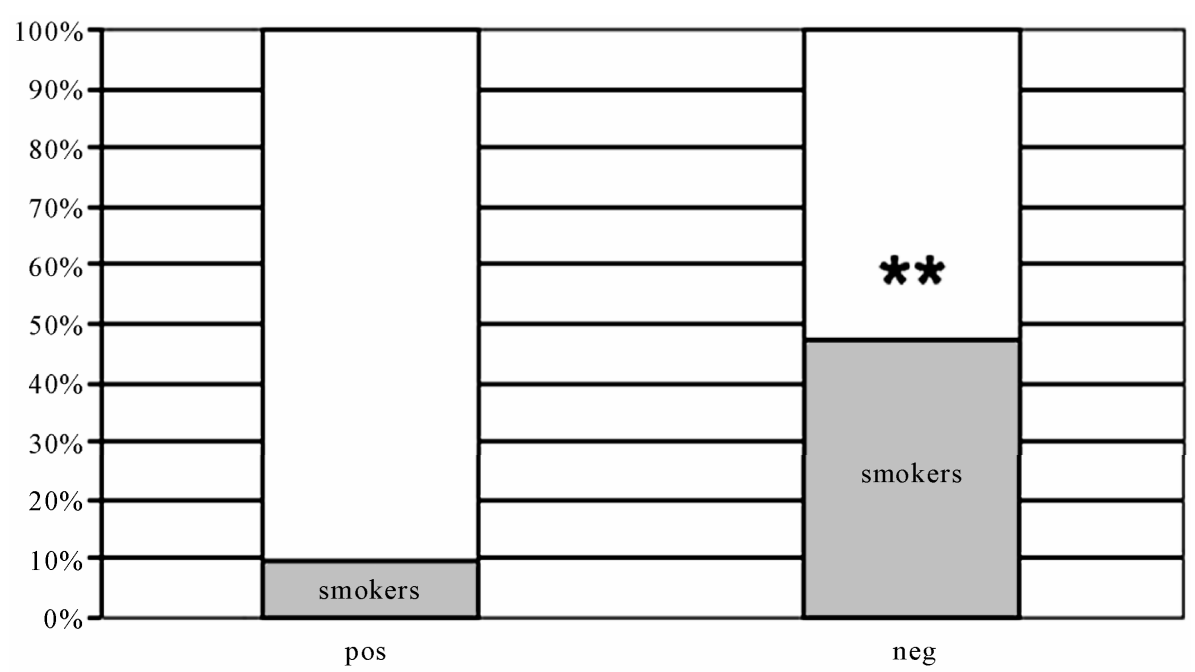

Figure 2. Ratio of smokers among endometriosis patients with regard to positive or negative outcome in self-image at the end of combined surgical and medical therapy. Data are presented as percentage values. ${ }^{* *} p<0.01$; pos, positive change in general quality of life; $n e g$, no or negative change in general quality of life. 
end of the study revealed no significant correlations.

\section{DISCUSSION}

The currently available medical treatments for endometriosis seem to be equally effective. It is estimated that about $80 \%-85 \%$ of patients have improvement in their symptoms [5]. Interestingly, severity of symptoms does not correlate well with the extent of the disease [3], although, in a well conducted study of 63 women a benefit from conservative surgery seemed to be greater in those with the most severe disease [8]. Unfortunately, there were only two participants in the group with severe disease, so limited data might hinder us to draw firm conclusion. In general, we know little about possible environmental and individual factors that can negatively influence the efficacy of different therapeutic modalities.

The present paper deals with change in pelvic pain and quality of life outcomes during combined surgical and medical treatment period in endometriosis patients, in relation to certain individual factors that might influence the effectiveness of therapy. The instrument we used to evaluate efficacy was a detailed questionnaire filled out twice by eligible and consenting patients.

In our study, the overall rate of improvement in quality of life was identified in almost three quarters of the final study cohort (73.7\%), and this is in line with other data [5]. Similarly to earlier observations [2,3], we could not find any correlation between the revealed extent of the disease and its impact on personal quality of life and pain scores reported by the patients. Moreover, no significant relation could be identified between these study end points and sociodemographic variables, such as marital status, level of education, number of previous pregnancies and births.

However, we found a striking relation between smoker status and pain relief, as well as overall improvement of quality of life. Those who were regular smokers in our study reported significantly less improvement in these fields. An explanation to this finding could be provided by a relatively new hypothesis raising, that, dioxin, the most toxic of the organochlorines, is associated with an observed increase in endometriosis in the developed world [1]. Dioxins (2,3,7,8-tetrachlorodibenzo-p-dioxin; TCDD) and dioxin-like chemicals cause a large variety of pathologies including immune dysfunction, carcinogenesis, developmental and reproductive abnormalities. Most of these toxic effects are mediated by aryl hydrocarbon receptor (AhR, also called the dioxin receptor), a ligand-activated transcription factor [9]. Recent investigation demonstrated that cigarette smoke contains high levels of agonists for AhR and markedly activates the dioxin signaling pathway [10].

The association of endometriosis with organochlori- nes, specifically polychlorinated biphenyls (PCBs) that are not dioxinlike, was first reported from Germany [11]. Also, a letter from Belgian gynecologists suggested that the higher prevalence of endometriosis at infertility clinics in Belgium could be caused by the relatively high TCDD concentration in the Belgian population [12]. In 1976, an explosion in Seveso, Italy exposed the surrounding population to among the highest levels of TCDD recorded in humans. The "Seveso Women's Health Study" addressed the relation between TCDD exposure and endometriosis and found a doubled but statistically nonsignificant risk for endometriosis in women with higher serum TCDD levels [13]. In nude mice, Bruner and coworkers demonstrated an augmenting effect of TCDD on the development of arteficial endometrial lesions [14]. Also, in a rat model, it was shown that the environmental pesticide methoxychlor, which can be metabolized to a chemical with high affinity for the estrogen receptor, had the same ability as estrogen to promote the growth of endometrial implants [15]. Methoxychlor is an example of synthetic organochlorines, a large and complex group of synthetic organic compounds containing chlorine atoms. The presence of chlorine tends to make the chemicals more stable. In fact, certain organochlorines, such as dioxins are extremely persistent and bioaccumulative [1]. Although in a study by Wilson et al. it was demonstrated that the maximum daily exposure estimates of dioxins deriving from mainstream cigarette smoke are below the current WHO Tolerable Daily Intake range of 1 - $5 \mathrm{pg} / \mathrm{kg}$ bw/day [16], because of the highly bioaccumulative properties of the organochlorines we need to be cautious when interpreting these data.

More is known about the mechanism of toxicity of dioxin than of almost any other chemical. It binds to the Ah receptor, which functions as a ligand-activated transcription factor [9]. Dioxin disturbs homeostasis, it is a known human carcinogen and is toxic to multiple organ systems [17]. Dioxins are reproductive and developmental toxicants, as well as being neurotoxic and immunotoxic $[18,19]$. Cytokines such as tumor necrosis factor and interleukins (IL) $1 \beta$ and 6 have also been shown to be induced by dioxin exposure $[20,21]$. TCDD has been shown to be an immune suppressant in multiple systems and was also recently suggested to cause autoimmunity in a mouse model [22]. Moreover, TCDD suppresses T-cell-mediated B-cell responses. It also causes a block in T-cell maturation and is associated with thymic atrophy at high doses in all species investigated [1]. These mechanisms might all contribute to the development and maintenance of endometriosis.

In some studies endometriosis was inversely related to cigarette smoking, however the available data were in- 
sufficient to clarify this point [23-26]. Moreover, these studies had different end points and they did not focus on improvement in general condition and pain after therapy in endometriosis patients earlier reporting pelvic pain. Also, in the study by Matorras et al., only a trend to a protective effect of smoking was detected [26]. Missmer et al. observed a complex relation with cigarette smoking [27]. The rate of endometriosis was not linearly associated with past smoking dose. However, the relation with current smoking differed by case-infertility status. Among women who had never reported infertility, cigarette smoking was directly associated with risk. However, when cases were concurrently infertile, current smoking was associated with reduced risk.

Based on our results, we can conclude, that regular smoking might have a disadvantageous impact on the success rate of combined surgical and medical therapy for endometriosis related pelvic pain. Although there are several studies focusing on the patomechanism of endometriosis with regard to smoking, to our knowledge, this is the first report dealing with a possible correlation between therapy issues and smoking in this field. Even though our data passed strict statistical analyses, considering their limited amount, we consider this work as a preliminary one to initiate specially aimed international studies to further clarify the issue.

\section{REFERENCES}

[1] Birnbaum, L.S. and Cummings, A.M. (2002) Dioxins and endometriosis: A plausible hypothesis. Environmental Health Perspectives, 110, 15-21. doi:10.1289/ehp.0211015

[2] Garai, J., Molnar, V., Varga, T., Koppan, M., Torok, A. and Bodis, J. (2006) Endometriosis: Harmful survival of an ectopic tissue. Frontiers in Bioscience, 11, 595-619. doi:10.2741/1821

[3] Balasch, J., Creus, M., Fabregues, F., et al. (1996) Visible and non-visible endometriosis at laparoscopy in fertile and infertile women and in patients with chronic pelvic pain: A prospective study. Human Reproduction, 11, 387-391.

[4] Tinelli, A., Martignago, R., Vergara, D., Leo, G., Malvasi, A. and Tinelli, R. (2008) Endometriosis management: Workflow on genomics and proteomics and future biomolecular pharmacotherapy. Current Medicinal Chemistry, 15: 2099-2107. doi:10.2174/092986708785747571

[5] Prentice, A. (2001) Regular review: Endometriosis. British Medical Journal, 323, 93-95. doi:10.1136/bmj.323.7304.93

[6] McCann, S.E., Freudenheim, J.L., Darrow, S.L., Batt, R.E. and Zielezny, M.A. (1993) Endometriosis and body fat distribution. Obstetrics \& Gynecology, 82, 545-549. doi:10.1097/00006250-199310000-00014

[7] Batt, R.E., Buck, G.M. and Smith, R.A. (1997) Health and fertility outcomes among women surgically treated for endometriosis. The Journal of the American Association of Gynecologic Laparoscopists, 4, 435-442.
doi:10.1016/S1074-3804(05)80035-0

[8] Sutton, C.J., Ewen, S.P., Whitelaw, N. and Haines, P. (1994) Prospective, randomized, double-blind, controlled trial of laser laparoscopy in the treatment of pelvic pain associated with minimal, mild, and moderate endometriosis. Fertility and Sterility, 62, 696-700.

[9] Birnbaum, L.S. (1994) Evidence for the role of the Ah receptor in response to dioxin. In: Spitzer, H.L., Slaga, T.J., Greenlee, W.F. and McClain, M., Ed., Receptor-Mediated Biological Processes: Implications for Evaluating Carcinogenesis, Progress in Clinical and Biological Research, 387, Wiley-Liss, New York, 139-54.

[10] Kitamura, M. and Kasai, A. (2007) Cigarette smoke as a trigger for the dioxin receptor-mediated signaling pathway. Cancer Letters, 252, 184-194. doi:10.1016/j.canlet.2006.11.015

[11] Gerhard, I. and Runnebaum, B. (1992) The limits of hormone substitution in pollutant exposure and fertility disorders. Zentralblatt für Gynäkologie, 114, 593-602.

[12] Koninckx, P.R., Braet, P., Kennedy, S.H. and Barlow, D.H. (1994) Dioxin pollution and endometriosis in Belgium. Human Reproduction, 9, 1001-1002.

[13] Eskenazi, B., Mocarelli, P., Warner, M., et al. (2002) Serum dioxin concentrations and endometriosis: A cohort study in Seveso, Italy. Environmental Health Perspectives, 110, 629-634. doi:10.1289/ehp.02110629

[14] Bruner, K.L., Matrisian, L.M., Rodgers, W.H., Gorstein, F. and Osteen, K.G. (1997) Suppression of matrix metalloproteinases inhibits establishment of ectopic lesions by human endometrium in nude mice. The Journal of Clinical Investigation, 99, 2851-2857. doi:10.1172/JCI119478

[15] Cummings, A.M. and Metcalf, J.L. (1995) Effects of estrogen, progesterone, and methoxychlor on surgically induced endometriosis in rats. Fundamental and Applied Toxicology, 27, 287-290. doi:10.1006/faat.1995.1135

[16] Wilson, C.L., Bodnar, J.A., Brown, B.G., Morgan, W.T., Potts, R.J. and Borgerding, M.F. (2008) Assessment of dioxin and dioxin-like compounds in mainstream smoke from selected US cigarette brands and reference cigarettes. Food and Chemical Toxicology, 46, 1721-1733. doi:10.1016/j.fct.2008.01.009

[17] IARC Working Group (1997) The evaluation of carcinogenic risks to humans: Polychlorinated dibenzo-paradioxins and polychlorinated dibenzofurans. IARC Monographs on the Evaluation of Carcinogenic Risks to Humans, 69, 1-631.

[18] Birnbaum, L.S. and Tuomisto, J. (2000) Non-carcinogenic effects of TCDD in animals. Food Additives \& Contaminants, 17, 275-288. doi:10.1080/026520300283351

[19] Yonemoto, J. (2000) The effects of dioxin on reproduction and development. Industrial Health, 38, 259-268. doi:10.2486/indhealth.38.259

[20] Lai, Z.W., Pineau, T. and Esser, C. (1996) Identification of dioxin-responsive elements (DREs) in the 5' regions of putative dioxin-inducible genes. Chemico-Biological Interactions, 100, 97-112. doi:10.1016/0009-2797(96)03691-5

[21] Lai, Z.W., Hundeiker, C., Gleichmann, E. and Esser, C. (1997) Cytokine gene expression during ontogeny in murine thymus on activation of the aryl hydrocarbon receptor by 2,3,7,8-tetrachlorodibenzo-p-dioxin. Molecular Pharmacology, 52, 30-37. 
[22] Holladay, S.D. (1999) Prenatal immunotoxicant exposure and postnatal autoimmune disease. Environmental Health Perspectives, 107, 687-691.

[23] Baron, J.A. (1996) Beneficial effects of nicotine and cigarette smoking: The real, the possible and the spurious. British Medical Bulletin, 52, 58-73.

[24] Cramer, D.W., Wilson, E., Stillman, R.J., et al. (1986) The relation of endometriosis to menstrual characteristics, smoking, and exercise. Journal of the American Medical Association, 255, 1904-1908. doi:10.1001/jama.255.14.1904

[25] Darrow, S.L., Vena, J.E., Batt, R.E., Zielezny, M.A., Michalek, A.M. and Selman, S. (1993) Menstrual cycle characteristics and the risk of endometriosis. Epidemiology, 4, 135-142.

doi:10.1097/00001648-199303000-00009

[26] Matorras, R., Rodiquez, F., Pijoan, J.I., Ramon, O., De Gutierrez, T.G., Rodriguez-Escudero, F. (1995) Epidemiology of endometriosis in infertile women. Fertility and Sterility, 63, 4-8.

[27] Missmer, S.A., Hankinson, S.E., Spiegelman, D., Barbieri, R.L., Marshall, L.M. and Hunter, D.J. (2004) Incidence of laparoscopically confirmed endometriosis by demographic, anthropometric, and lifestyle factors. American Journal of Epidemiology, 160, 784-796. doi:10.1093/aje/kwh275 